\title{
Understanding the Resistance Mechanism in Brassica napus to Clubroot Caused by Plasmodiophora brassicae
}

\author{
Jiaqin Mei, ${ }^{1,2}$ Zhen Guo, ${ }^{3}$ Jinhua Wang, ${ }^{1,2}$ Yuxia Feng, ${ }^{1,2}$ Guanhua Ma, ${ }^{3}$ Chunyu Zhang, ${ }^{4}$ Wei Qian, ${ }^{1,2}$ and \\ Guokang Chen ${ }^{3, \dagger}$ \\ ${ }^{1}$ College of Agronomy and Biotechnology, Southwest University, Chongqing 400716, China; ${ }^{2}$ Academy of Agricultural Sciences, Southwest \\ University, Chongqing, China; ${ }^{3}$ College of Plant Protection, Southwest University, Chongqing 400716, China; and ${ }^{4}$ College of Plant Science \\ \& Technology, Huazhong Agricultural University, Wuhan, 430070, China \\ Accepted for publication 24 December 2018.
}

ABSTRACT

\begin{abstract}
Exploring the mechanism of plant resistance has become the basis for selection of resistance varieties but reports on revealing resistant mechanism in Brassica napus against Plasmodiophora brassicae are rare. In this study, RNA-seq was conducted in the clubroot-resistant B. napus breeding line ZHE-226 and in the clubroot-susceptible rapeseed cultivar Zhongshuang 11 at $0,3,6$, 9, and 12 days after inoculation. Strong alteration was detected specifically in ZHE-226 as soon as the root hair infection happened, and significant promotion was found in ZHE-226 on cell division or cell cycle, DNA repair and synthesis, protein synthesis, signaling, antioxidation, and secondary metabolites. Combining results from physiological, biochemical, and histochemical assays, our study highlights an effective signaling in
\end{abstract}

ZHE-226 in response to $P$. brassicae. This response consists of a fast initiation of receptor kinases by $P$. brassicae; the possible activation of host intercellular $\mathrm{G}$ proteins which might, together with an enhanced $\mathrm{Ca}^{2+}$ signaling, promote the production of reactive oxygen species; and programmed cell death in the host. Meanwhile, a strong ability to maintain homeostasis of auxin and cytokinin in ZHE-226 might effectively limit the formation of clubs on host roots. Our study provides initial insights into resistance mechanism in rapeseed to P. brassicae.

Keywords: Brassica napus; clubroot; Plasmodiophora brassicae; resistance mechanism; signaling network; transcriptome sequencing.
The soilborne obligate plant pathogen Plasmodiophora brassicae causes clubroot in many Brassica plants, including rapeseed (Brassica napus L.), an economically important crop worldwide (Dixon 2009; Neik et al. 2017; Ren et al. 2016; Strelkov and Hwang 2014). During infection, $P$. brassicae initially infects root hairs, then the root cortex, leading to root swelling and gall formation. These galls hinder the uptake of water and nutrients and result in abnormal shoot growth (Kageyama et al. 2009). The pathogen can survive in soil for several years; thus, clubroot is difficult to control with pesticides. Therefore, developing resistant cultivars is the most effective and sustainable way to manage clubroot.

Understanding the defense response and molecular mechanism in a host against a pathogen is of great significance in the breeding of resistant varieties. Proteomic and transcriptome studies were conducted in Arabidopsis, B. rapa, B. oleracea, and B. juncea, revealing many factors associated with mechanisms of resistance to $P$. brassicae in these host species. Among these are cell wall modifications, cell cycle alterations, the production of secondary metabolites, calcium and hormone signaling and homeostasis, the activation of pathogen-associated molecular patterns, the activity of effector receptors (resistance genes) and pathogenesis-related (PR)

†Corresponding author: G. Chen; E-mail: chenguokang@swu.edu.cn

Funding: This study was financially supported by the National Key R\&D Program of China (2017YFD0201100), the Science and Technology Innovation Program for the Social Undertakings and the People's Livelihood in Chongqing (cstc2015shmsztzx80011, cstc2016shmsx0674, and cstc2016shmszx80074), and the Fundamental Research Funds for the Central Universities (XDJK2018B022 and XDJK2018AA004).

J. Mei and Z. Guo contributed equally to this work.

*The $\boldsymbol{e}$-Xtra logo stands for "electronic extra" and indicates that one supplementary table is published online.

The author(s) declare no conflict of interest.

(c) 2019 The American Phytopathological Society genes, the induction of transcriptional factors and mitogenactivated protein kinases, or the formation of reactive oxygen species (ROS) (Chen et al. 2016; Irani et al. 2018; Luo et al. 2018; Siemens et al. 2006; Zhang et al. 2016; Zhao et al. 2017). However, these provided limited reference values for understanding the resistance mechanism of rapeseed because no transcriptome study has been reported in rapeseed, mainly due to the lack of resistant resources in B. napus. Among the six Brassica spp. in U's triangle (Nagaharu 1935), innate clubroot resistance (CR) is found frequently in B. rapa (particular turnip), B. oleracea, and B. nigra (Carlsson et al. 2004; Crisp et al. 1989; Hasan et al. 2012; Manzanares-Dauleux et al. 2000) but rarely in B. napus. On the other hand, genetic studies have revealed different modulation patterns of resistance genes or loci on CR in B. napus (possibly in quantitative manner) (Neik et al. 2017; Piao et al. 2009; Rahman et al. 2014) and in B. rapa (qualitative plus quantitative manner) (Piao et al. 2002; Sakamoto et al. 2008; Suwabe et al. 2006). Therefore, studies in rapeseed are necessary to understand its resistance mechanism against $P$. brassicae.

Recently, a Chinese B. napus variety, Huashuang 5R, exhibited high resistance to the most prevalent pathotype of $P$. brassicae in China (pathotype 4) (Zhan 2017). In order to understand the response in clubroot-resistant $B$. napus to $P$. brassicae, transcriptomic, physiological, biochemical, and histochemical assays were conducted in the present study between a susceptible B. napus genotype and a resistant genotype ZHE-226 that carries the same CR locus (PbBa8.1) with Huashuang 5R (Zhan 2017). Our data revealed great differences between the two rapeseed genotypes in response to $P$. brassicae, particularly on activation of signaling networks and modulation of ROS and programmed cell death (PCD).

\section{MATERIALS AND METHODS}

Plant materials and $\boldsymbol{P}$. brassicae inoculation. A Chinese rapeseed variety, $B$. napus 'Zhongshuang 11', and rapeseed breeding line ZHE-226 were assessed for disease severity for 2 
years in a clubroot-infested field in Fuling, Chongqing, China (E107.6459, N29.5709). Disease index (DI) was measured at the flowering stage according to the method of Ashizawa et al. (1980). Meanwhile, the resistance was investigated under controlledenvironment condition in lab according to the liquid inoculation technique (Luo et al. 2014). Briefly, resting spores isolated from root galls (pathotype 4) were diluted to a density of $10^{7}$ spores $\cdot \mathrm{ml}^{-1}$ in sterile distilled water. Roots of each 4-day-old plant were soaked in the spore suspension in a $15-\mathrm{ml}$ plastic tube and maintained in a growth chamber under a 16 -h photoperiod at $25^{\circ} \mathrm{C}$ for 20 days. Then, plants were transplanted into soil in 10-by-10-cm pots and rated for DI according to Ashizawa et al. (1980) 40 days after transplanting. Three biological replications were conducted, with 30 plants in each genotype inoculated.

Monitor of dynamic infection in roots. The dynamic infection processes of $P$. brassicae in the roots of two rapeseed genotypes were monitored using an inverted microscope (Olympus IX70) every $12 \mathrm{~h}$ after inoculation until secondary infection was established. The roots were stabilized in Carnoy's solution (methanol/acetone, 3:1) for $24 \mathrm{~h}$ at room temperature, then washed three to five times with distilled water, stained with $0.5 \%$ phloxine, and observed under a microscope. Based on observation, the root hair infection and cortical infection started at 3 and 6 days after inoculation (dai), respectively. Therefore, the ratio of infected root hairs was investigated in 100 root hairs per plant every 2 days between 3 and 15 dai, and the average number of infected cortices on roots was counted from three microscopic fields per plant at 6 (the starts of Zhongshuang 11), 7 (the starts of ZHE-226), 9, 11, 13, and 15 dai. Three independent biological replicates were conducted, with three plants observed per experimental unit.

Test of ROS concentration. The ROS concentration was investigated in the roots of two genotypes at 3, 6, 9, 12, and 15 dai with the Plant ROS ELISA Kit (Shanghai Renjie Biotechnology, China) according to the manufacturer's instructions. In brief, supernatant was prepared after $20 \mathrm{~min}$ of centrifugation $(3,000 \mathrm{rpm})$ of phosphate-buffer-( $\mathrm{pH} 7.2)$-ground roots from each single plant. Each supernatant $(50 \mu \mathrm{l})$ was incubated at $37^{\circ} \mathrm{C}$ for $60 \mathrm{~min}$ with $100 \mu \mathrm{l}$ of horseradish peroxidase (HRP) conjugate reagent in a well that contained HRP-labeled antibody. Liquid was then discarded and each well was washed with washing buffer five times. Chromogen solution $(100 \mu \mathrm{l})$ was added into each well and kept at $37^{\circ} \mathrm{C}$ under darkness for $15 \mathrm{~min}$, and absorbance at $450 \mathrm{~nm}$ was measured within 15 min after the adding $50 \mu$ l of elimination agent. ROS concentration was calculated from the absorbance of each sample according to the standard sample curve. In total, three biological replicates were conducted, with eight samples measured in each repetition.

Activity analysis of antioxidant enzymes. The activity of antioxidant enzymes, including catalase (CAT), superoxide dismutase (SOD), and peroxidase (POD), was investigated in the roots of two genotypes at 3,6, 9, 12, and 15 dai according to Gao (2006). Three biological replicates were carried out, with five technical replicates per root sample. Briefly, root homogenate was prepared in $5 \mathrm{ml}$ of phosphate buffer $(0.05 \mathrm{~mol} / \mathrm{liter}, \mathrm{pH} 7.0)$. For CAT, the reaction was initiated by adding $0.2 \mathrm{ml}$ of $\mathrm{H}_{2} \mathrm{O}_{2}(0.2 \mathrm{~mol} / \mathrm{liter}), 1 \mathrm{ml}$ of Tris- $\mathrm{HCl}(0.05 \mathrm{~mol} /$ liter, $\mathrm{pH} 7.0)$, and $1.7 \mathrm{ml}$ of distilled water into $2 \mathrm{ml}$ of root homogenate. The decrease in optical density due to the decomposition of $\mathrm{H}_{2} \mathrm{O}_{2}$ was measured at the end of 1 min against blanks (roots homogenate was replaced by phosphate buffer) at $240 \mathrm{~nm}$. A unit of CAT was defined as the amount of enzyme that decomposed $\mathrm{H}_{2} \mathrm{O}_{2}$ at $1 \mu \mathrm{M} / \mathrm{min}$. The specific activity was expressed in terms of units per gram of roots. For SOD, root homogenate $(0.1 \mathrm{ml})$ was taken, and $0.3 \mathrm{ml}$ of methionine $(\mathrm{Met})(130 \mathrm{mM})$, $0.3 \mathrm{ml}$ of nitroblue tetrazolium (NBT) $(750 \mu \mathrm{mol} / \mathrm{liter})$, and $0.3 \mathrm{ml}$
A

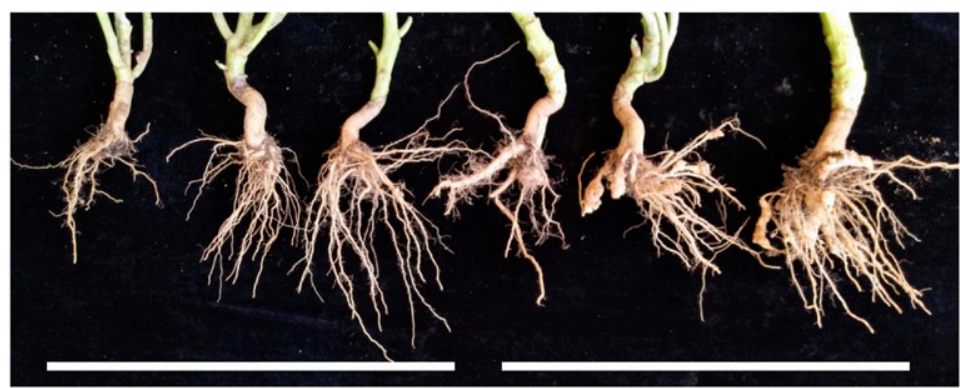

Z-226

ZS 11
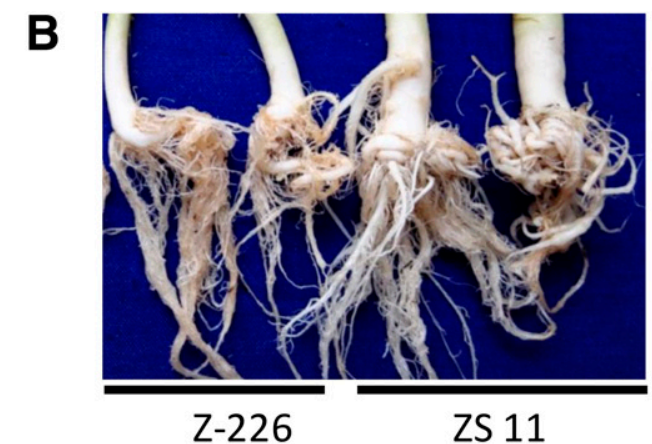

Z-226
ZS 11
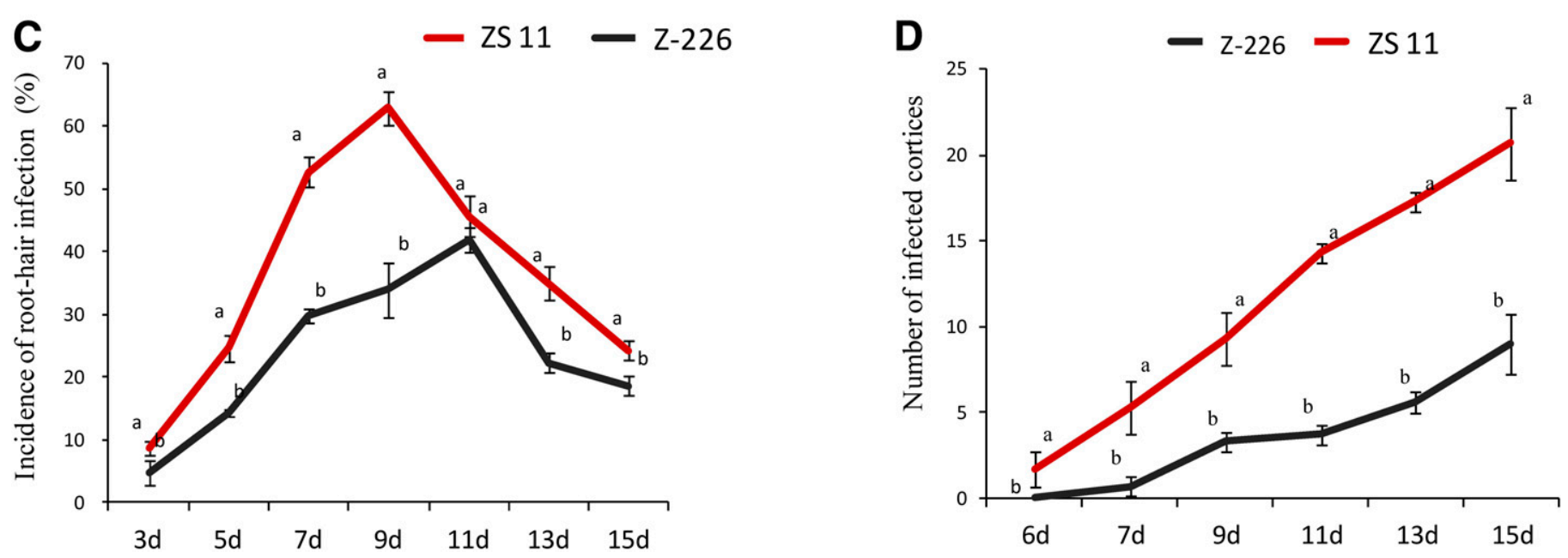

Fig. 1. Difference on clubroot resistance between ZHE-226 (Z-226) and Zhongshuang 11 (ZS11). A and B, Root performance of two lines after inoculation with

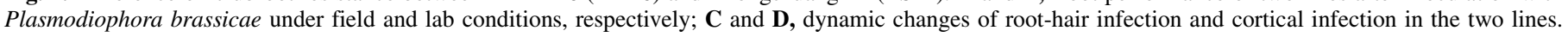


of EDTA- $\mathrm{Na}_{2}(0.1 \mathrm{mmol} / \mathrm{liter})$ were added. The reaction was initiated by adding $0.3 \mathrm{ml}$ of lactoflavine ( $20 \mu \mathrm{mol} / \mathrm{liter})$. Zero time absorbance was taken at $560 \mathrm{~nm}$ followed by recording the absorbance after $20 \mathrm{~min}$ at $25^{\circ} \mathrm{C}$. The control was simultaneously run without root homogenate. A unit of SOD activity was defined as the amount of enzyme required to inhibit the reduction of NBT by $50 \%$. The specific activity was expressed in terms of units per milligram of fresh root. For SOD, root homogenate $(0.5 \mathrm{ml})$ was taken, and $1 \mathrm{ml}$ of potassium iodide (PI) solution (10 mmol/liter) and $1 \mathrm{ml}$ of sodium acetate solution $(40 \mathrm{mmol} / \mathrm{liter})$ were added. The absorbance of potassium periodide was read at $353 \mathrm{~nm}$, which indicated the amount of POD. Further, $20 \mu \mathrm{l}$ of $\mathrm{H}_{2} \mathrm{O}_{2}(15 \mathrm{mmol} / \mathrm{liter})$ was added, and the change in the absorbance was recorded after $5 \mathrm{~min}$. A unit of POD activity was defined as the amount of enzyme required to change optical density at $1 \mathrm{U} / \mathrm{min}$. The specific activity was expressed as units per milligram of fresh root.

Histochemical staining. Apoptosis and necrosis were visualized with Hoechst and propidium iodide (PI) at 3, 6, 9, 12, 15, and 20 dai. In detail, roots were soaked in phosphate-buffered saline (PBS) solution $(0.05 \mathrm{~mol} / \mathrm{liter}, \mathrm{pH} 7.8)$ containing $5 \%$ Hoechst and $5 \%$ PI for $30 \mathrm{~min}$ at $4^{\circ} \mathrm{C}$ and washed with PBS solution before being viewed with a fluorescence microscope (Olympus).

Transcriptome sequencing. The roots from 10 plants were sampled separately in Zhongshuang 11 and ZHE-226 at 0, 3, 6, 9,

A

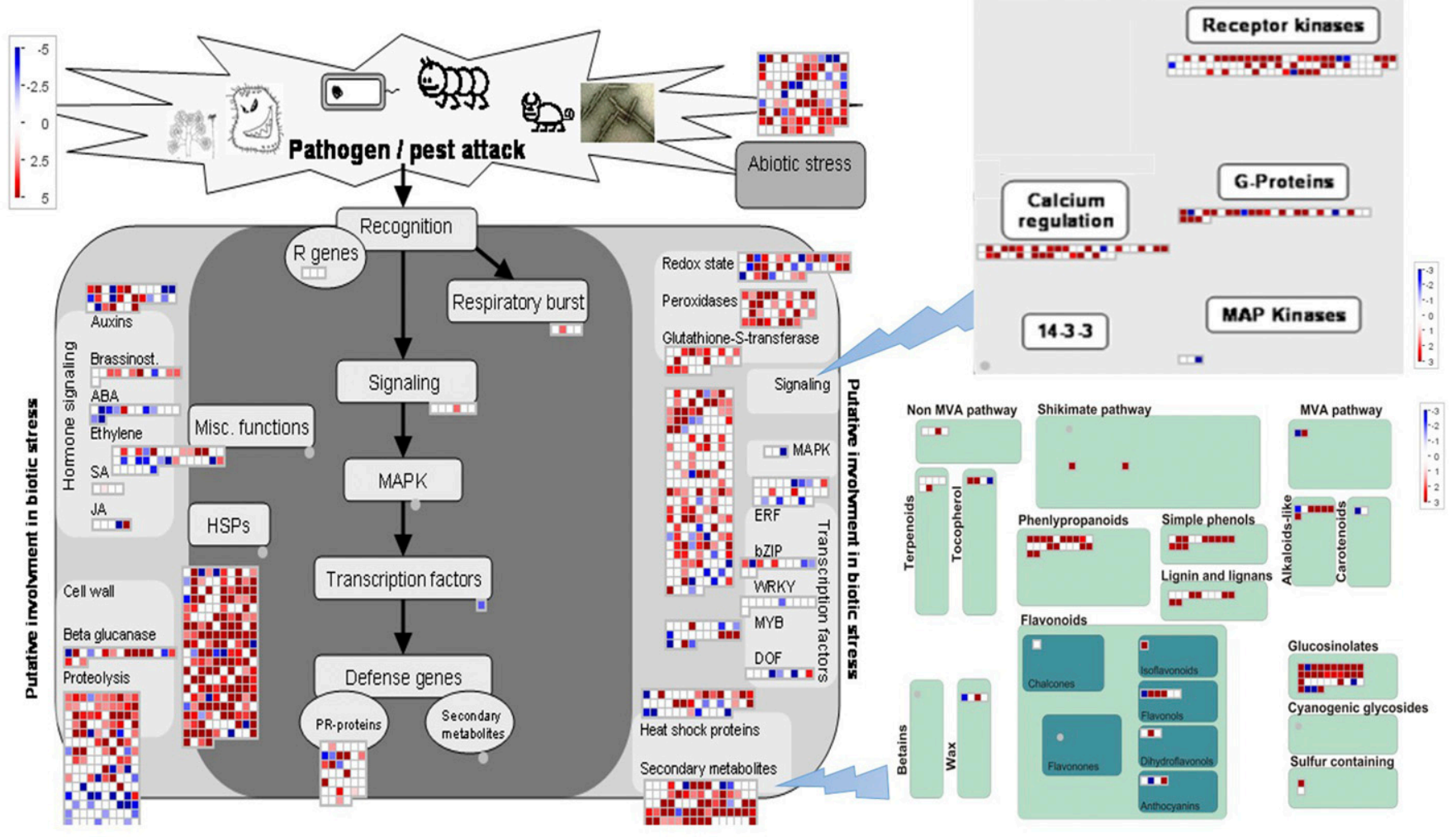

B

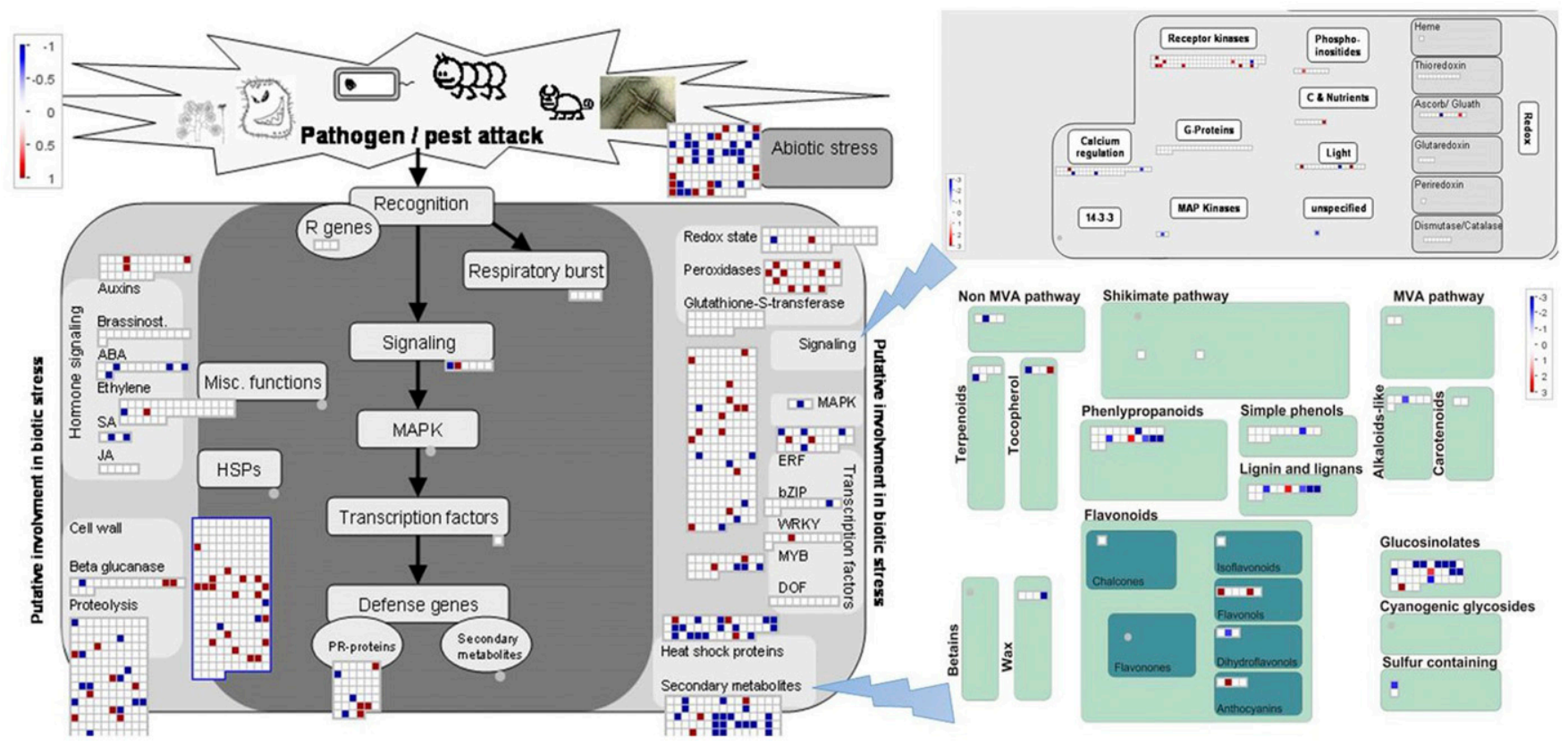

Fig. 2. Analyses of biotic stress associated pathways in A, ZHE-226 and B, Zhongshuang 11 at 3 days after inoculation by Plasmodiophora brassicae. 
and 12 dai. Three independent biological replicates were performed and the roots were pooled in each genotype according to time point. Total RNA was extracted using TRNzol-A+Reagent (TianGen) and 10 library preparations were sequenced on an Illumina Hiseq 2000 platform. The sequences were base called and quality checked by the Illumina data-processing pipeline. Raw reads were filtered to obtain high-quality clean reads by removing adaptor sequences, duplicated sequences, reads containing more than $5 \%$ "N" (i.e., ambiguous bases in reads), and reads in which more than $50 \%$ of the bases showed a $Q$ value $\leq 5$. Clean reads were aligned to the B. napus genome sequence (https://www.ncbi.nlm.nih.gov/ genome/203?genome_assembly_id=335272) using SOAP2 (http:// soap.genomics.org.cn/). Uniquely mapped reads were used for gene expression analysis. Abundances of all unigenes were estimated using reads per kilobyte per million mapped reads (Mortazavi et al. 2008). The threshold determining the significance of differentially expressed genes (DEGs) among multiple tests was set at a false discovery rate $\leq 0.001$ and $\mid \log _{2}$ (fold change) $\mid \geq 1$ (Audic and Claverie 1997; Dang et al. 2013). Functional enrichment analyses were performed using MAPMAN software (http://mapman.gabipd. org/) based on the $\log _{2}$ (fold change) of DEGs, and pathway frames presented in this article were derived from the MAPMAN software database (Thimm et al. 2004). A heatmap was constructed by the software "heatmap.2." according to the $\log _{2}$ (fold change) of interested DEGs (Deng et al. 2014).

\section{RESULTS}

Resistance performance. DI ratings and root observations confirm that infection by $P$. brassicae in Zhongshuang 11 was earlier and more severe than in ZHE-226. Significant difference in DI between two genotypes was detected under both the lab and the field conditions (Fig. 1), with $\mathrm{DI}_{Z \text { hongshuagn11-field }}=80$, $\mathrm{DI}_{\text {ZHE226-field }}=0, \mathrm{DI}_{\text {Zhongshuagn11-lab }}=46$, and $\mathrm{DI}_{\text {ZHE226-lab }}=10$. According to the infection process of $P$. brassicae monitored in two genotypes, root-hair infection was observed in both lines at 3 dai, while cortical infection occurred in Zhongshuang 11 at 6 dai, which was 1 day earlier than that in the resistant genotype. Incidence of infected root hairs and number of infected cortices were both higher in Zhongshuang 11 than in ZHE-226 (Fig. 1C and D).

Transcriptome sequencing and expression alterations. According to the dynamics of early infection, transcriptome sequencing was conducted in ZHE-226 and Zhongshuang 11 at 0 , 3 (root-hair infection), 6 (cortical infection), 9 (peak of root-hair infection), and 12 dai. Approximately $6.99 \mathrm{~Gb}$ clean reads were generated from each root sample, of which 66.9 to $71.6 \%$ were aligned to the reference genome of B. napus. The GC content from 10 libraries ranged from 46.3 to $47.7 \%$ and the Q30\% values were all $>90 \%$. In total, 67,383 unigenes were detected from the 10 root samples, of which 7,319 genes were identified as DEGs between neighboring time points. In total, 5,071 DEGs were detected from ZHE-226, while only 3,435 were found from Zhongshuang 11, with 1,270 overlap genes. ZHE-226 exhibited a decrease of DEG number along with the infection time, whereas Zhongshuang 11 showed an increase of DEGs during 3 to 9 dai (Supplementary Table S1). The data suggest a faster and stronger response to $P$. brassicae in ZHE226 as compared with that in Zhongshuang 11.

Overview on cell function alterations. In order to understand general alterations on cell function, DEGs were analyzed in MAPMAN, which displays large data sets in detailed diagrams of metabolic pathways or processes. Upregulations were detected in ZHE-226 for a majority of pathways at 3 dai, with a slight decrease at subsequent time points, whereas many pathways and processes in Zhongshuang 11 were mainly suppressed at 6 and 9 dai. During 0 to 3 dai, the period exhibiting significant difference between ZHE-226 and Zhongshuang 11, substantial transcriptional changes were detected in ZHE-226 on cell division or cell cycle, DNA repair and synthesis, protein synthesis, cell organization, and redox, with a majority of DEGs upregulated. In contrast, only a few genes were changed in Zhongshuang 11, functionally associated with biotic or
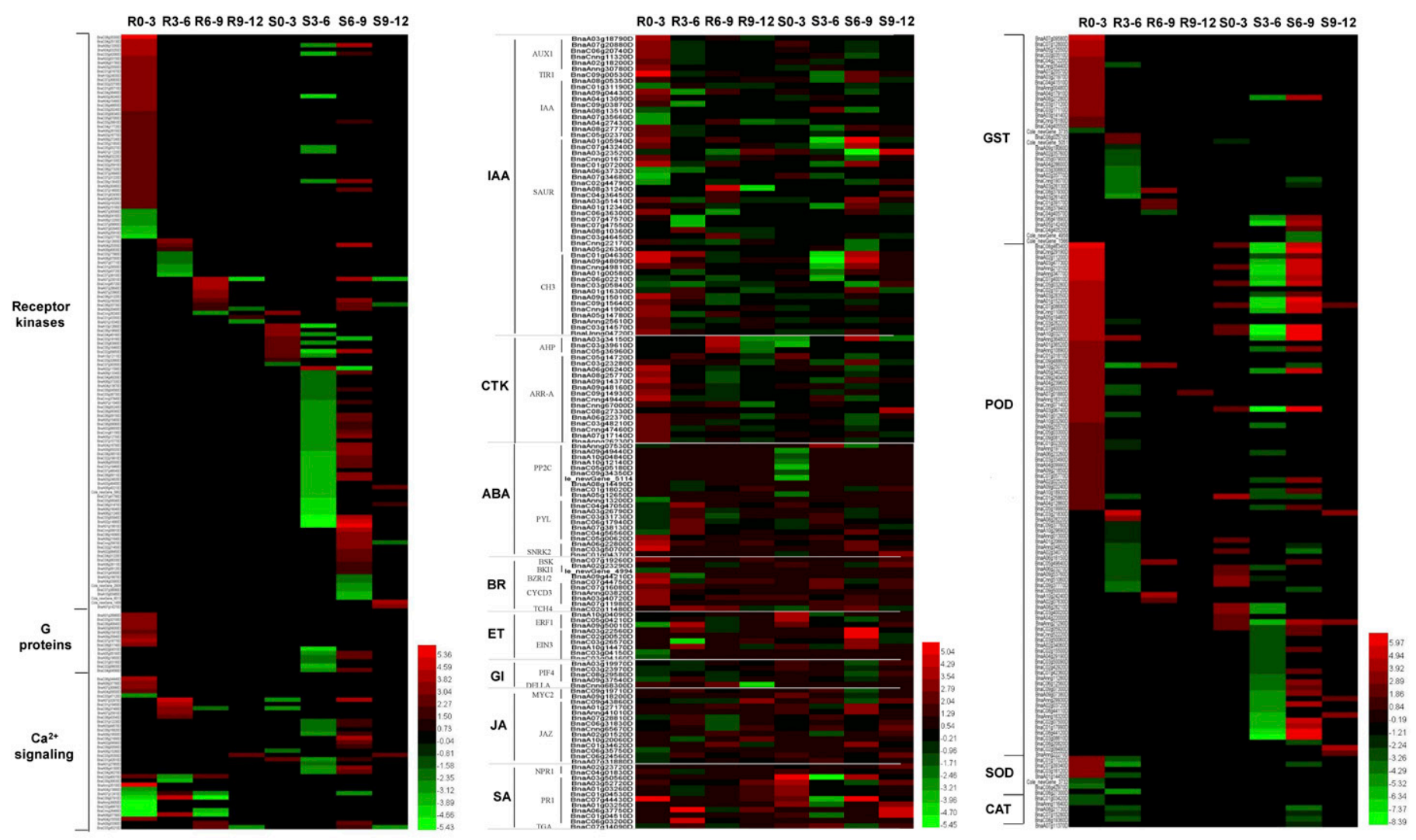

Fig. 3. Heatmap of differentially expressed genes of interest in ZHE-226 (R) and Zhongshuang 11 (S) according to the $\log _{2}$ (fold change) values. 
abiotic stress (irregularly altered) and amino acid activation (downregulated).

Alterations on biotic stress associated processes. Great differences were detected between the two genotypes on processes associated with biotic response in the whole infection duration, particularly during 0 to 3 dai. DEGs were mainly upregulated in ZHE-226 at 3 dai, with the majority promoting processes involved in cell wall, signaling, antioxidation (redox state, peroxidases, and glutathione-S-transferase), and secondary metabolites (particularly glucosinolates, phenlypropanoids, simple phenols, lignin, lignans, and alkaloides) (Fig. 2). Comparatively, Zhongshuang 11 presented significant suppression of signaling, antioxidation, and secondary metabolites (Fig. 2). Considering the great difference between the two genotypes on signaling and antioxidation, and further considering the close association of these two aspects with ROS and PCD, detailed analyses and experiments were conducted and results were presented as follows.

Signaling. A significant difference was detected between the two genotypes in expression profiles of receptor kinases, $\mathrm{G}$ proteins, $\mathrm{Ca}^{2+}$ signaling, and hormone-mediated signal transduction (Fig. 3). Receptor kinases are protein molecules receiving signals from outside cells. Expressions of 41 receptor kinases were immediately upregulated at 3 dai in ZHE-226 and constantly expressed during the entire infection period. In contrast, 10 receptors were activated in Zhongshuang 11 at 3 dai, including 6 exhibiting temporary upregulations. Furthermore, 21 receptor kinases were suppressed in Zhongshuang 11 at 6 dai. The data indicate that the inducement of receptor kinases by $P$. brassicae is fast and strong in the resistant genotype, and that these kinases are suppressed in the susceptible genotype.

$\mathrm{G}$ proteins and their associates such as GTP-binding nuclear proteins and guanine nucleotide exchange factors are involved in transmitting signals from a variety of stimuli outside a cell to its interior. In total, 14 DEGs in this category were detected in this study, of which 8 were activated 5- to 16-fold in ZHE-226 from 3 dai on, while 6 were transiently suppressed 5- to 11 -fold in Zhongshuang 11 during 3 to 6 dai (Fig. 3). This suggested the fast and constant activation of $\mathrm{G}$ proteins and their associates in the resistant rapeseed after inoculation.

Upregulation of genes involved in $\mathrm{Ca}^{2+}$ signaling, an important cellular signaling in defense response, was detected in ZHE-226 at 3 dai (Fig. 3). In detail, eight genes encoding calcium-binding proteins (CMLs) and cyclic nucleotide-gated ion channels (CNGCs) were induced in ZHE-226 immediately after inoculation, and five CMLs were additionally activated at 6 dai. In contrast, 11 DEGs involved in $\mathrm{Ca}^{2+}$ signaling were downregulated in Zhongshuang 11 during 0 to 6 dai, except for an upregulation on one gene. These suggest an activation of $\mathrm{Ca}^{2+}$ signaling in ZHE-226 after inoculation and suppression in Zhongshuang 11.

Different expression patterns were detected between the two genotypes for hormone-mediated signal transductions, including auxin-, cytokinin (CTK)-, abscisic acid-, brassinosteroid-, gibberellin-, ethylene-, jasmonic acid-, and salicylic acid-mediated signal transduction pathways (Fig. 3). Most obviously, opposite regulation patterns of auxin- and CTK-mediated signal transductions were detected between the two genotypes, particularly during 0 to 3 dai. Specifically, 39 DEGs involved in the auxin-mediated signal transduction pathway were detected from ZHE-226 during the infection, of which 26 were upregulated immediately after inoculation (3 dai), and 19 DEGs were detected in Zhongshuang 11 during 6 to 12 dai, with only 5 genes induced by the pathogen. Similarly, 18 genes involved in the CTK-mediated signal transduction pathway were altered in ZHE-226 during infection, with 13 genes encoding A-ARRs upregulated at 3 dai and 3 genes encoding AHPs activated at 6 dai, whereas 9 DEGs were detected from the susceptible genotype, with only 2 genes upregulated before 6 dai. These suggest a fast and strong inducement of auxin- and CTKmediated signal transductions in the resistant genotype in response to the pathogen.

ROS and PCD. Various signals are associated with activation of ROS, which contributes to suppress the fungi growth and regulates host PCD and hypersensitive reaction. Three $R B O H \mathrm{~s}$, the key factors in initiation of ROS, were significantly activated 15 -fold in ZHE-226 from 3 dai on, while four $R B O H$ s were transiently suppressed 6- to 14-fold in Zhongshuang 11 during 6 to 9 dai. To confirm the difference between the two genotypes, the ROS concentration was investigated in roots during 0 to 15 dai (Fig. 4). Compared with the uninoculated controls, the resistant genotype ZHE-226 exhibited elevated ROS levels, particularly during 6 to 12 dai, whereas ROS was heavily and constantly suppressed in the susceptible genotype before 15 dai. In addition, apoptosis (the typical form of PCD) and necrosis were checked by staining technique in roots of the two genotypes during 0 to 20 dai, which detected obvious apoptosis during 6 to 20 dai in ZHE-226 without
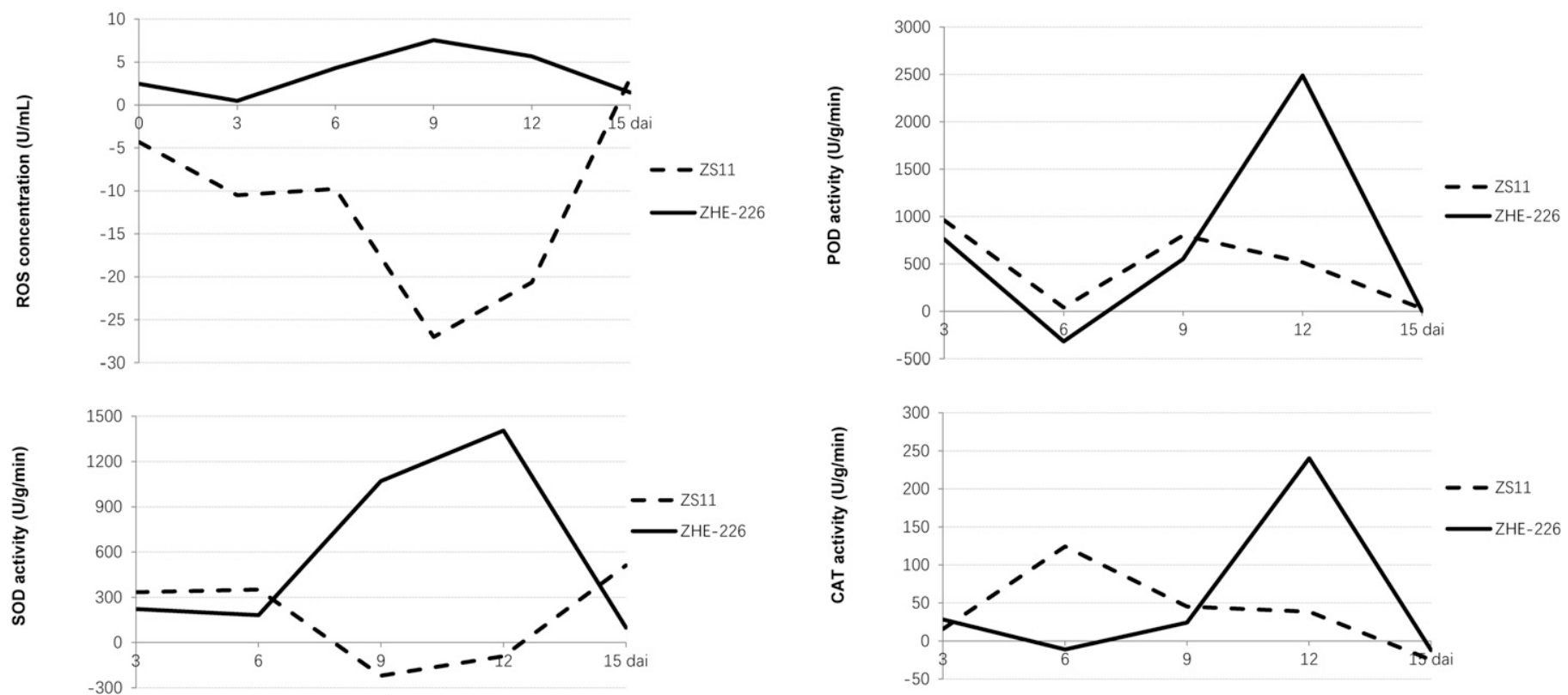

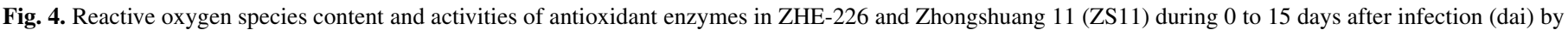
Plasmodiophora brassicae. 
obvious necrosis (Fig. 5). Comparatively, light apoptosis was found in Zhongshuang 11, in particular during 9 to 20 dai, with necrotic cells visible at 15 dai and serious necrosis (loss cell shapes) at 20 dai (Fig. 5).

Antioxidation. A significant difference was detected between the two genotypes in expression profiles of genes encoding antioxidant enzymes, including glutathione- $S$-transferase, POD, CAT, and SOD (Fig. 3). In total, 70 such genes were upregulated in ZHE-226, of which 58 were constantly increased for expression during the whole infection period. By contrast, 19 antioxidant genes were upregulated in Zhongshuang 11 at 3 dai, including 14 temporary activations (heavily repressed at 6 dai). Furthermore, 53 antioxidant genes were downregulated in Zhongshuang 11 at 6 and 9 dai. A biochemical study was conducted in the two genotypes to confirm their difference in antioxidating ability. Significant increases of enzyme activity of POD, CAT, and SOD were detected uniquely in ZHE-226 at 12 dai (Fig. 4), suggesting a higher antioxidating ability in the resistant genotype as compared with the susceptible rapeseed.

\section{DISCUSSION}

Defense responses in $B$. rapa, B. oleracea, B. juncea, and Arabidopsis against $P$. brassicae have been previously reported to be associated with many factors, of which the most often mentioned pathways were cell wall enforcement, biosynthesis of secondary metabolites (glucosinolates, lignins, terpenoids, proanthocyanidins, and so on), calcium homeostasis, hormone signaling, and ROS generation or detoxication (Cao et al. 2008; Chen et al. 2016; Irani et al. 2018; Luo et al. 2018; Siemens et al. 2006; Zhang et al. 2016; Zhao et al. 2017). Because different host species, different genotypes (some studies used only susceptible genotypes), and different time points were studied, it is difficult to compare our DEGs with these studies, except Chen et al. (2016) and Zhang et al. (2016), who studied the responses of both resistant and susceptible genotypes before gall formation stage (see the following paragraphs for details). Nevertheless, our transcriptomic, physiological, biochemical, and histochemical assays revealed fast (as early as the beginning of root-hair infection) and strong activation in the resistant B. napus genotype ZHE-226 of the aforementioned processes. Moreover, our study seems to indicate an effective signaling network in the resistant rapeseed in response to P. brassicae (Fig. 6).

In the present study, strong activation of receptor kinases and $\mathrm{G}$ proteins was detected, specifically in ZHE-226 at the beginning of root hair infection, consistent with previous studies (Chen et al. 2016; McDonald et al. (2014). McDonald et al. (2014) indicated
A

R-CK
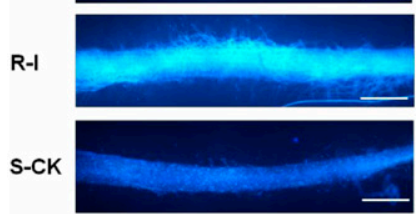

S-I

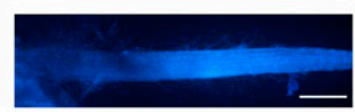

6
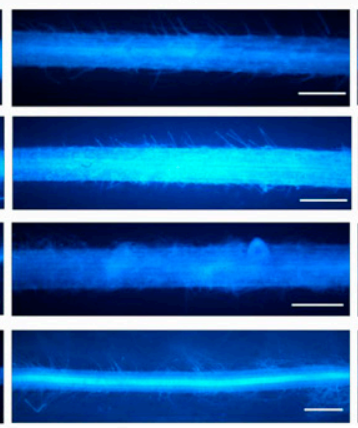

9

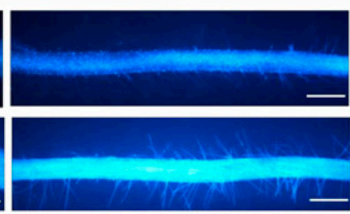

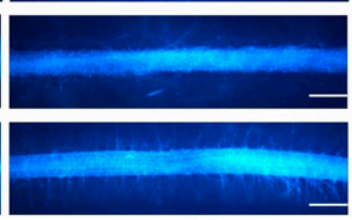

12
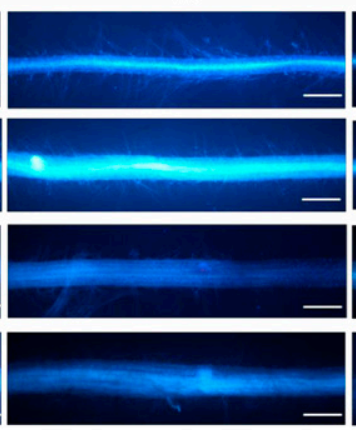

15 dai
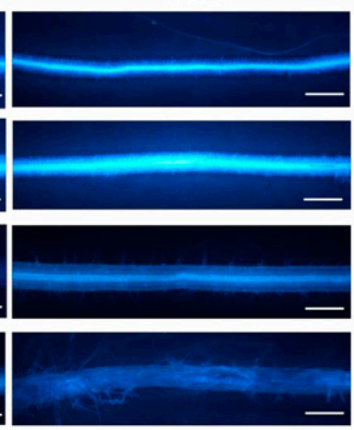

B

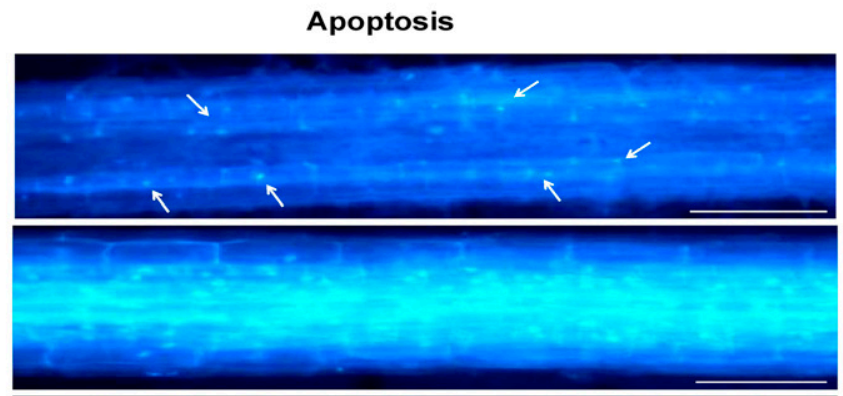

S-CK 20
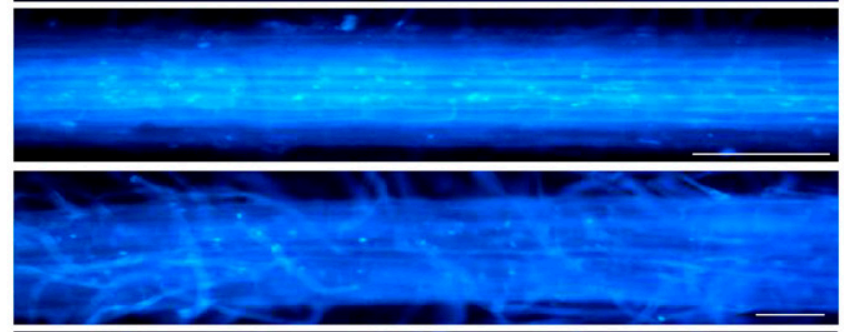

S-I 15

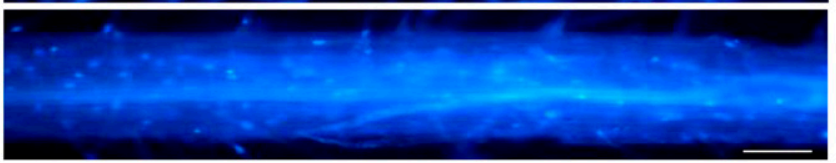

Necrosis
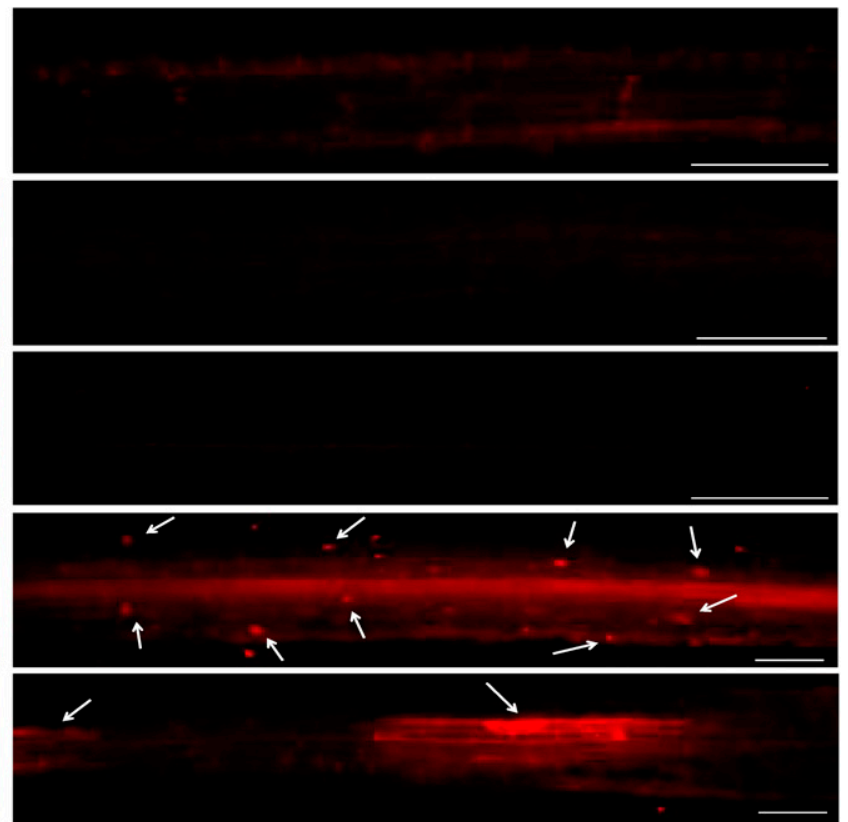

Fig. 5. Apoptosis and cell death in ZHE-226 (R) and Zhongshuang $11(\mathrm{~S})$ during infection by Plasmodiophora brassicae. ROS $=$ reactive oxygen species, POD = peroxidase, $\mathrm{SOD}=$ superoxide dismutase, and CAT = catalase. A, Apoptosis in roots of two rapeseed genotypes during 0 to 15 days after inoculation (dai); $\mathbf{B}$, apoptosis and necrosis in roots of two rapeseed genotypes at 15 and 20 dai. Uninoculated controls (CK) and infected (I) roots were stained simultaneously with Hoechst and propidium iodide for apoptosis (arrows show the typical apoptotic cells) and necrosis (indicated by arrows), respectively. Photos of apoptosis and necrosis were taken from the same position of root for each sample. Bars $=100 \mu \mathrm{m}$. 
that the recognition of $P$. brassicae by $B$. napus occurs during roothair infection. Chen et al. (2016) found that expression levels of effector receptors (resistance proteins) were higher in clubrootresistant $B$. rapa near-isogenic lines (NILs) during 12 to $96 \mathrm{~h}$ after inoculation as compared with the susceptible NIL. Receptors are protein molecules receiving signals from outside a cell and relaying, amplifying, or integrating signals (Alberts et al. 2014; Hall 2016); the $G$ protein-coupled receptors (GPCRs) formed the largest family of receptors (Hall 2016). GPCRs spanning the cell membrane activate $G$ proteins (including heterotrimeric $G$ proteins and small GTPases) within the cell, which route the outside signals to intracellular signaling pathways (Neves et al. 2002). Studies have revealed that small GTPases were involved in $\mathrm{H}_{2} \mathrm{O}_{2}$ production, PCD, and defense-gene expression in plants (Kawasaki et al. 1999; Moeder et al. 2005; Ono et al. 2001; Suharsono et al. 2002). It was also reported that small GTPases could regulate the activity of auxin efflux transporter PIN2, Aux/IAAs, or ARFs to modulate the auxin signal transduction (Li et al. 2005; Tao et al. 2002, 2005). Therefore, the fast and strong activation of receptors and $G$ proteins in ZHE-226 after infection may trigger transmission of a pathogenperception signal to intracellular signaling pathways such as ROS, PCD, and hormone signal transduction.
Intracellular calcium homeostasis and $\mathrm{Ca}^{2+}$ signaling were reported to associate with the resistance in hosts to $P$. brassicae (Cao et al. 2008; Chen et al. 2016; Luo et al. 2018; Zhang et al. 2016). Similarly, we detected fast initiation of $\mathrm{Ca}^{2+}$ signaling in the resistant rapeseed after inoculation, including two CML23 genes (BnaA07g25810D and BnaC06g43540D) which are homologs to one DEG detected in B. rapa (Bra004165) (Chen et al. 2016) and four genes (BnaC06g34440D, BnaA07g30990D, BnaA09g41300D, and BnaC04g36270D), homologs to two CNGC14 and two CML26, detected in $B$. oleracea after inoculation with $P$. brassicae (Zhang et al. 2016). The $\mathrm{Ca}^{2+}$ signal activates a downstream signaling cascade and generates secondary messengers such as ROS (Bindschedler et al. 2001; Takahashi et al. 2011), which contribute to induced host PCD and suppressed fungi growth (Clarke et al. 2000). Under the $\mathrm{Ca}^{2+}$ efflux, in addition, a small GTPase was reported to induce $\mathrm{PR}$ gene expression, $\mathrm{H}_{2} \mathrm{O}_{2}$ production, and $\mathrm{PCD}$ (Moeder et al. 2005). Consistently, we detected upregulation of ROS-associated genes, including one $R B O H A$ and two $R B O H C$, which were also activated in resistant $B$. olerace $a$ after inoculation with $P$. brassicae (Zhang et al. 2016). Furthermore, biochemical study revealed strong ROS and PCD in ZHE-226 in response to $P$. brassicae. On the other hand, to avoid ROS-caused damage

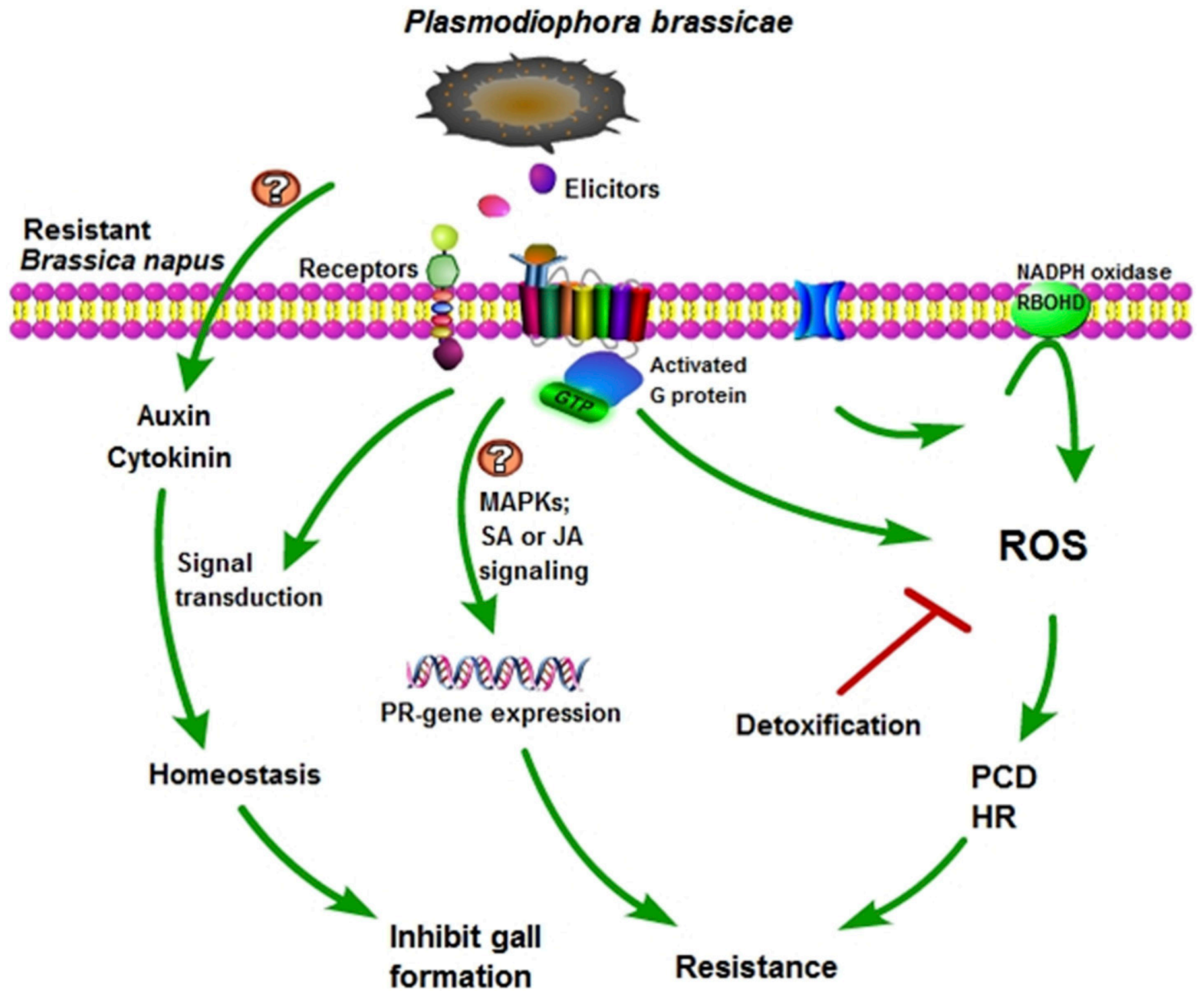

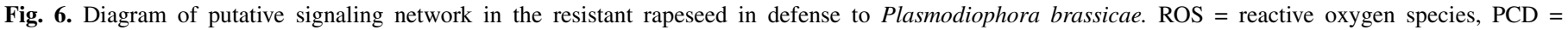
programmed cell death, $\mathrm{HR}=$ hypersensitive reaction, MAPK = mitogen-activated protein kinase, $\mathrm{SA}=$ salicylic acid, and JA = jasmonic acid. 
and cell death in the host, antioxidant enzymes were effectively activated in ZHE-226 at 12 dai when a high level of ROS was accumulated. Therefore, the enhanced $\mathrm{Ca}^{2+}$ signaling and activated small GTPases in the resistant genotype possibly promoted the ROS and PCD and, thus, suppressed the infection and limited the expansion of $P$. brassicae; in addition, the effective ROS scavenging system of the resistant rapeseed possibly helped to alleviate the damage on host cells.

Although there are issues regarding the roles of auxin and CTK in the interaction between host and P. brassicae (possibly due to species differences and time points differences), clubroot symptoms were reported to be correlated with the increase of auxin, particularly during early infection (Ludwig-Müller et al. 1993, 1996; Siemens et al. 2006), and it seems that the CTK content of clubbed Brassica spp. increased during the early infection stage (Dekhuijzen and Overeem 1971; Devos et al. 2005, 2006; LudwigMüller 2014; Siemens et al. 2006) but possibly was suppressed during the gall formation stage (Malinowski et al. 2016). In the present study, we detected an increase in auxin metabolism in the resistant genotype because the auxin signal transduction pathway was significantly activated in ZHE-226. In addition, four nitrilases whose up expressions were found to synthesize more auxin during the development of a club (Grsic-Rausch et al. 2000; Siemens et al. 2006) were downregulated 9- to18-fold in ZHE-226 from 3 dai on, while two nitrilases were activated up to 41 -fold in the susceptible genotype at 3 dai (data not shown). Similarly, the CTK signal transduction pathway was significantly activated in the resistant genotype at 3 dai. Moreover, three oxidases and dehydrogenases $(C K X \mathrm{~s})$, which are responsible for CTK degradation and exhibit a positive effect on resistance to P. brassicae (Siemens et al. 2006), were suppressed five- to sixfold in the susceptible genotype, while a $C K X 3$ was upregulated sixfold in ZHE-226 (data not shown). These data suggest that, compared with the susceptible genotype, the resistant rapeseed possibly decreases the accumulation of auxin and CTK during the early infection stage via repressing the synthesis, activating the metabolism, or promoting the degradation of these hormones.

\section{LITERATURE CITED}

Alberts, B., Bray, D., Hopkin, K., Johnson, A. D., Lewis, J., Raff, M., Roberts, K., and Walter, P. 2014. Essential Cell Biology, 4th ed. Garland Science, New York, NY, U.S.A.

Ashizawa, M., Yoshikawa, H., and Hida, K. 1980. Studies on breeding for resistance to clubroot in Brassica. II. Screening of cole crops for clubrootresistance. Bull. Veg. Ornam. Crops Res. Stn. A 7:35-75.

Audic, S., and Claverie, J. M. 1997. The significance of digital gene expression profiles. Genome Res. 7:986-995.

Bindschedler, L. V., Minibayeva, F., Gardner, S. L., Gerrish, C., Davies, D. R., and Bolwell, G. P. 2001. Early signalling events in the apoplastic oxidative burst in suspension cultured French bean cells involve cAMP and $\mathrm{Ca}^{2+}$. New Phytol. 151:185-194.

Cao, T., Srivastava, S., Rahman, M. H., Kav, N. N. V., Hotte, N., Deyholos, M. K., and Strelkov, S. E. 2008. Proteome-level changes in the roots of Brassica napus as a result of Plasmodiophora brassicae infection. Plant Sci. 174:97-115.

Carlsson, M., Von, B. R., and Merker, A. 2004. Screening and evaluation of resistance to downy mildew (Peronospora parasitica) and clubroot (Plasmodiophora brassicae) in genetic resources of Brassica oleracea. Hereditas 141:293-300.

Chen, J., Pang, W., Chen, B., Zhang, C., and Piao, Z. 2016. Transcriptome analysis of Brassica rapa near-isogenic lines carrying clubroot-resistant and -susceptible alleles in response to Plasmodiophora brassicae during early infection. Front. Plant Sci. 6:1183.

Clarke, A., Desikan, R., Hurst, R. D., Hancock, J. T., and Neill, S. J. 2000. NO way back: Nitric oxide and programmed cell death in Arabidopsis thaliana suspension cultures. Plant J. 24:667-677.

Crisp, P., Crute, I. R., Sutherland, R. A., Angell, S. M., Bloor, K., Burgess, H., and Gordon, P. L. 1989. The exploitation of genetic resources of Brassica oleracea in breeding for resistance to clubroot (Plasmodiophora brassicae). Euphytica 42:215-226.

Dang, Z. H., Zheng, L. L., Wang, J., Gao, Z., Wu, S. B., Qi, Z., and Wang, Y. C. 2013. Transcriptomic profiling of the salt-stress response in the wild recretohalophyte Reaumuria trigyna. BMC Genomics 14:29.
Dekhuijzen, H. M., and Overeem, J. C. 1971. The role of cytokinins in clubroot formation. Physiol. Mol. Plant 1:151-161.

Deng, W., Wang, Y., Liu, Z., Cheng, H., and Xue, Y. 2014. HemI: A toolkit for illustrating heatmaps. PLoS One 9:e111988.

Devos, S., Laukens, K., Deckers, P., Van Der Straeten, D., Beeckman, T., Inzé, D., Van Onckelen, H., Witters, E., and Prinsen, E. 2006. A hormone and proteome approach to picturing the initial metabolic events during Plasmodiophora brassicae infection on Arabidopsis. Mol. Plant-Microbe In. 19: 1431-1443.

Devos, S., Vissenberg, K., Verbelen, J. P., and Prinsen, E. 2005. Infection of Chinese cabbage by Plasmodiophora brassicae leads to a stimulation of plant growth: Impacts on cell wall metabolism and hormone balance. New Phytol. 166:241-250.

Dixon, G. R. 2009. The occurrence and economic impact of Plasmodiophora brassicae and clubroot disease. J. Plant Growth Regul. 28:194-202.

Gao, J. 2006. Experimental Guidance for Plant Physiology. Higher Education Press, Beijing, China.

Grsic-Rausch, S., Kobelt, P., Siemens, J. M., Bischoff, M., and Ludwig-Müller, J. 2000. Expression and localization of nitrilase during symptom development of the clubroot disease in Arabidopsis. Plant Physiol. 122:369-378.

Hall, J. 2016. Guyton and Hall Textbook of Medical Physiology. Elsevier Saunders, Philadelphia, PA, U.S.A.

Hasan, M. J., Strelkov, S. E., Howard, R. J., and Rahman, H. 2012. Screening of Brassica germplasm for resistance to Plasmodiophora brassicae pathotypes prevalent in Alberta, Canada. Can. J. Plant Sci. 92:501-515.

Irani, S., Trost, B., Waldner, M., Nayidu, N., Tu, J., Kusalik, A. J., Todd, C. D., Wei, Y., and Bonhamsmith, P. C. 2018. Transcriptome analysis of response to Plasmodiophora brassicae infection in the Arabidopsis shoot and root. BMC Genomics 19:23.

Kageyama, K., Asano, T., and Dixon, G. R. 2009. Life cycle of Plasmodiophora brassicae. J. Plant Growth Regul. 28:203-211.

Kawasaki, T., Henmi, K., Ono, E., Hatakeyama, S., Iwano, M., Satoh, H., and Shimamoto, K. 1999. The small GTP-binding protein Rac is a regulator of cell death in plants. Proc. Natl. Acad. Sci. U.S.A. 96:10922-10926.

Li, L., Xu, J., Xu, Z. H., and Xue, H. W. 2005. Brassinosteroids stimulate plant tropisms through modulation of polar auxin transport in Brassica and Arabidopsis. Plant Cell 17:2738-2753.

Ludwig-Müller, J. 2014. Auxin homeostasis, signaling, and interaction with other growth hormones during the clubroot disease of Brassicaceae. Plant Signal. Behav. 9: Article e28593.

Ludwig-Müller, J., Bendel, U., Thermann, P., Ruppel, M., Epstein, E., and Hilgenberg, W. 1993. Concentrations of indole-3-acetic acid in plants of tolerant and susceptible varieties of Chinese cabbage infected with Plasmodiophora brassicae Woron. New Phytol. 125:763-769.

Ludwig-Müller, J., Epstein, E., and Hilgenberg, W. 1996. Auxin-conjugate hydrolysis in Chinese cabbage: Characterization of an amidohydrolase and its role during infection with clubroot disease. Physiol. Plant. 97: 627-634.

Luo, H. C., Chen, G. K., Liu, C. P., Huang, Y., and Xiao, C. G. 2014. An improved culture solution technique for Plasmodiophora brassicae infection and the dynamic infection in the root hair. Australas. Plant Pathol. 43:53-60.

Luo, Y., Dong, D., Su, Y., Wang, X., Peng, Y., Peng, J., and Zhou, C. 2018. Transcriptome analysis of Brassica juncea var. tumida Tsen responses to Plasmodiophora brassicae primed by the biocontrol strain Zhihengliuella aestuarii. Funct. Integr. Genomics 18:301-314.

Malinowski, R., Novák, O., Borhan, M. H., Spíchal, L., Strnad, M., and Rolfe, S. A. 2016. The role of cytokinins in clubroot disease. Eur. J. Plant Pathol. 145:543-557.

Manzanares-Dauleux, M. J., Divaret, I., Baron, F., and Thomas, G. 2000. Evaluation of French Brassica oleracea land races for resistance to Plasmodiophora brassicae. Euphytica 113:211-218.

McDonald, M. R., Sharma, K., Gossen, B. D., Deora, A., Feng, J., and Hwang, S. F. 2014. The role of primary and secondary infection in host response to Plasmodiophora brassicae. Phytopathology 104:1078-1087.

Moeder, W., Yoshioka, K., and Klessig, D. F. 2005. Involvement of the small GTPase Rac in the defense responses of tobacco to pathogens. Mol. PlantMicrobe Interact. 18:116-124.

Mortazavi, A., Williams, B. A., McCue, K., Schaeffer, L., and Wold, B. 2008. Mapping and quantifying mammalian transcriptomes by RNA-Seq. Nat. Methods 5:621-628.

Nagaharu, U. 1935. Genome analysis in Brassica with special reference to the experimental formation of B. napus and peculiar mode of fertilization. Jpn. J. Bot. 7:389-452.

Neik, T. X., Barbetti, M. J., and Batley, J. 2017. Current status and challenges in identifying disease resistance genes in Brassica napus. Front. Plant Sci. 8:1788.

Neves, S. R., Ram, P. T., and Iyengar, R. 2002. G Protein Pathways. Science 296:1636-1639. 
Ono, E., Wong, H. L., Kawasaki, T., Hasegawa, M., Kodama, O., and Shimamoto, K. 2001. Essential role of the small GTPase Rac in disease resistance of rice. Proc. Natl. Acad. Sci. U.S.A. 98:759-764.

Piao, Z., Ramchiary, N., and Lim, Y. P. 2009. Genetics of clubroot resistance in Brassica species. J. Plant Growth Regul. 28:252-264.

Piao, Z. Y., Park, Y. J., Choi, S. R., Hong, C. P., Park, J. Y., Choi, Y. S., and Lim, Y. P. 2002. Conversion of an AFLP marker linked to clubroot resistance gene in Chinese cabbage into a SCAR marker. J. Korean Soc. Hortic. Sci. 43:653-659.

Rahman, H., Peng, G., Yu, F., Falk, K. C., Kulkarni, M., and Selvaraj, G. 2014. Genetics and breeding for clubroot resistance in Canadian spring canola (Brassica napus L.). Can. J. Plant Pathol. 36:122-134.

Ren, L., Xu, L., Liu, F., Chen, K., Sun, C., Li, J., and Fang, X. 2016. Host range of Plasmodiophora brassicae on cruciferous crops and weeds in China. Plant Dis. 100:933-939.

Sakamoto, K., Saito, A., Hayashida, N., Taguchi, G., and Matsumoto, E. 2008. Mapping of isolate-specific QTLs for clubroot resistance in Chinese cabbage (Brassica rapa L. ssp. pekinensis). Theor. Appl. Genet. 117:759-767.

Siemens, J., Keller, I., Sarx, J., Kunz, S., Schuller, A., Nagel, W., Schmülling, T., Parniske, M., and Ludwig-Müller, J. 2006. Transcriptome analysis of Arabidopsis clubroots indicate a key role for cytokinins in disease development. Mol. Plant-Microbe Interact. 19:480-494.

Strelkov, S. E., and Hwang, S. F. 2014. Clubroot in the Canadian canola crop: 10 years into the outbreak. Can. J. Plant Pathol. 36:27-36.

Suharsono, U., Fujisawa, Y., Kawasaki, T., Iwasaki, Y., Satoh, H., and Shimamoto, K. 2002. The heterotrimeric G protein $\alpha$ subunit acts upstream of the small GTPase Rac in disease resistance of rice. Proc. Natl. Acad. Sci. U.S.A. 99:13307-13312.

Suwabe, K., Tsukazaki, H., Iketani, H., Hatakeyama, K., Kondo, M., Fujimura, M., Nunome, T., Fukuoka, H., Hirai, M., and Matsumoto, S. 2006. Simple sequence repeat-based comparative genomics between Brassica rapa and Arabidopsis thaliana: The genetic origin of clubroot resistance. Genetics 173:309-319.

Takahashi, F., Mizoguchi, T., Yoshida, R., Ichimura, K., and Shinozaki, K. 2011. Calmodulin-Dependent Activation of MAP Kinase for ROS Homeostasis in Arabidopsis. Mol. Cell 41:649-660.

Tao, L., Cheung, A. Y., and Wu, H. 2002. Plant Rac-like GTPases are activated by auxin and mediate auxin-responsive gene expression. Plant Cell 14: 2745-2760.

Tao, L. Z., Cheung, A. Y., Nibau, C., and Wu, H. M. 2005. RAC GTPases in tobacco and Arabidopsis mediate auxin-induced formation of proteolytically active nuclear protein bodies that contain AUX/IAA proteins. Plant Cell 17:2369-2383.

Thimm, O., Bläsing, O., Gibon, Y., Nagel, A., Meyer, S., Krüger, P., Selbig, J., Müller, L. A., Rhee, S. Y., and Stitt, M. 2004. MAPMAN: A user-driven tool to display genomics data sets onto diagrams of metabolic pathways and other biological processes. Plant J. 37:914-939.

Zhan, Z. 2017. Clubroot resistant germplasm innovation and transferring of resistant locus $\mathrm{PbBa} 8.1$ from turnip into canola elite variety. Huazhong Agricultural University, Wuhan, China (in Chinese with English abstract).

Zhang, X., Liu, Y., Fang, Z., Li, Z., Yang, L., Zhuang, M., Zhang, Y., and Lv, H. 2016. Comparative transcriptome analysis between broccoli (Brassica oleracea var. italica) and wild cabbage (Brassica macrocarpa Guss.) in response to Plasmodiophora brassicae during different infection stages. Front. Plant Sci. 7:1929.

Zhao, Y., Bi, K., Gao, Z., Chen, T., Liu, H., Xie, J., Cheng, J., Fu, Y., and Jiang, D. 2017. Transcriptome analysis of Arabidopsis thaliana in response to Plasmodiophora brassicae during early infection. Front. Microbiol. 8: 673 